\title{
Measurement of self-shaped ellipsoidal bunches from a photoinjector with postacceleration
}

\author{
Brendan O'Shea* and James B. Rosenzweig \\ Department of Physics and Astronomy, University of California at Los Angeles, Los Angeles, California 90095, USA
}

Galina Asova, Jürgen Bähr, Marc Hänel, Yevgeniy Ivanisenko, Martin Khojoyan, Mikhail Krasilnikov, Lazar Staykov, and Frank Stephan

Deutsches Elektronen Synchrotron DESY, Platanenallee 6, 15738 Zeuthen, Germany

Juliane Rönsch-Schulenburg

Universität Hamburg, Luruper Chaussee 149, 22761 Hamburg, Germany

(Received 3 August 2010; published 10 January 2011)

\begin{abstract}
Recent work has shown the possibility of generating self-shaped ellipsoidal beams with properties commensurate with the requirements of future light sources such as free-electron lasers and inverse Compton sources. In this so-termed "blowout" regime, short laser bunches are transformed via photoemission into short electron bunches which then self-consistently evolve into nearly uniform-density ellipsoids under space-charge forces. We report here on the first blowout studies conducted in collaboration between the UCLA Particle Beam Physics Lab and the Photo Injector Test Facility, Zeuthen (PITZ). The measurements conducted at the PITZ photoinjector facility examine the evolution of $750 \mathrm{pC}, 2.7 \mathrm{ps}$ FWHM electron bunches born in an L-band photoinjector and subsequently accelerated through a ninecell L-band booster for a resulting energy of $12 \mathrm{MeV}$. These measurements represent the first observations of self-shaped ellipsoid evolution under postinjector acceleration, a key step in demonstrating the utility of such self-shaped beams at higher energy, where the advantages in both transverse and longitudinal and transverse phase space may be exploited in creating very high brightness beams.
\end{abstract}

DOI: 10.1103/PhysRevSTAB.14.012801

PACS numbers: 29.27.Bd, 29.25.Bx, 41.75.Ht, 41.85.Ct

\section{INTRODUCTION}

The prevailing method for producing high brightness beams has been until recently a combination of the emittance compensation process [1,2] with a nearly uniformly filled cylindrical electron beam. The uniformly filled cylindrical electron beam is used in an attempt to create a beam whose space-charge forces are linear in distance from the axis. This trait is most suitable for the emittance compensation process, in which the transverse phase space distributions of different longitudinal slices of the beam may be approximated as thin lines of differing orientation in phase space; compensation refers to the realignment of these lines. Unfortunately, such a beam suffers from nonlinear space-charge fields in the head and the tail which are not well compensated, and thus result in emittance growth. Further, the longitudinal fields are highly nonlinear, particularly near the beam head and tail. These fields drive strong distortions to the initial "flattop" longitudinal beam profile. In contrast, a uniformly filled ellipsoidal beam distribution generates linear space-charge self-forces throughout the entire beam. This optimal beam for emittance preservation in both transverse and longitudinal senses has been in the literature for many decades, and

\footnotetext{
*Corresponding author. brendan.d.oshea@gmail.com
}

known for even longer [3-5], but until recently has been difficult to produce in the laboratory.

A novel approach to the creation of uniformly filled ellipsoidal beams was introduced by Serafini, where an initially short laser pulse was used to illuminate a cathode and the subsequently emitted electron beam was allowed to expand longitudinally under space-charge forces [6]. This idea was later expanded upon by Luiten et al. to include a more rigorous determination of the ideal radial profile of the laser for generation of a hard edge uniformly filled ellipsoid [7]. Work has continued on such a method of beam creation which has shown its compatibility with the emittance compensation process [8] and culminated in an exhaustive experimental characterization of a nearly uniform ellipsoidal beam $[9,10]$ directly from a photocathode electron gun at moderate $(3.5 \mathrm{MeV})$ energy.

Taking into account recent trends in accelerator designs that demand both high peak and average current for light source injectors, our goal for this experiment was twofold. First, and most importantly, we wished to examine the evolution of self-shaped beams after acceleration in a linac, where the final emittance compensation occurs [2]. This step is critical in the demonstration of the utility of using self-shaped beams in photoinjectors, as the acceleration process quickly diminishes the strength of the spacecharge forces that self-consistently maintain the ellipsoidal shape of the beam. In other words, at low energy, the fact 
that the six-dimensional phase space distribution is not of the highly particular (and very difficult to realize) Kapchinskii-Vladimirski form [3] is not important, due to the extreme dominance of space-charge forces over emittance effects [2]. At high enough energy, one may ignore transverse space-charge effects, but there must be a transition between these two limits. It is precisely this transition regime we study here. Further, acceleration serves to prepare the beam for the commonly employed technique of magnetic compression. It is asserted through theory and simulation that the (again) nearly linear form of the longitudinal phase space distribution greatly improves its compressibility [8]; this linear form has been observed directly at the exit of a photocathode gun [10]. The present work examines directly the combined effects of acceleration and longitudinal space charge on the quality of the longitudinal phase space.

Second, the interest that the free-electron laser (FEL) community displays in high average current pushes system designs towards very high duty factor operation. Thus, L-band photoinjectors and accelerators, based on superconducting or high average power normal conducting structures, are envisioned as key elements of such systems. As such, we seek to extend the study of the blowout regime to L-band structures, where the time scales involved in the beams are expanded with respect to those in S-band by over a factor of 2. This expansion of scope allows use of (appropriate to high average power designs) existing, relatively long-pulse laser technology. Further, we take advantage of beam creation and characterization techniques in a state-of-the-art high average current facility, the DESY Photo Injector Test Facility, Zeuthen (PITZ) for this study. The context has obvious application to future high power FELs, since PITZ functions as the test bed for the injector envisioned for the European XFEL project. We begin with a description of the experiment and the simulations leading to its design and follow with a presentation of data collected and a discussion of results and future work.

\section{EXPERIMENT}

The experiment was conducted at the PITZ photoinjector, a $1300 \mathrm{MHz}$ system consisting primarily of a photocathode rf gun, emittance compensation focusing solenoid, and postacceleration (booster) linac. In these experiments, a $2.7 \mathrm{ps}$ FWHM laser pulse was used to illuminate a $\mathrm{Cs}_{2} \mathrm{Te}$ photocathode in the L-band 1.6 cell rf gun. For this experiment both the gun and booster were run near rf crest and the gun itself was fed power appropriate to reach a peak onaxis field of $60 \mathrm{MV} / \mathrm{m}$. The beam is focused using the emittance compensation solenoid into the nine-cell L-band booster and accelerated to $12.25 \mathrm{MeV} / c$. The beam's emittance is measured using a single slit-scan technique $[11,12]$ and the longitudinal phase space is characterized using a dispersive arm $[13,14]$.
The PITZ laser system is capable of producing $257 \mathrm{~nm}$ light in an $800 \mu$ s pulse train, at an intratrain pulse repetition frequency of $1 \mathrm{MHz}$, with an overall system repetition rate of $10 \mathrm{~Hz}[15,16]$. These light pulses are used to photoexcite a $\mathrm{Cs}_{2} \mathrm{Te}$ cathode. The pulse profiles are measured using an optical sampling system for the longitudinal direction and a virtual cathode system for the transverse $[8,9]$. The laser pulse for the present experiments has the following characteristics: 2.7 ps FWHM (1.15 ps rms) temporal duration and is cut transversely using a $2 \mathrm{~mm}$ diameter beam shaping aperture resulting in a $0.47 \mathrm{~mm} \mathrm{rms}$ truncated transverse Gaussian producing a charge of $750 \mathrm{pC}$ off the cathode. This transverse shape approximates well the optimum "half-circle" distribution that produces the nearly uniformly filled ellipsoidal distributions [7] through the blowout process. This launched distribution was studied using PARMELA simulations to give a strong self-field-driven expansion (nontrivial selfshaping), while minimizing the deleterious effects, discussed below, of the image charge at the cathode.

In order to characterize the most relevant aspects of the beam dynamics in this regime, two central measurements of beam distribution are needed: the longitudinal phase space, and the transverse phase space and associated emittance. The dispersive section used to measure the longitudinal phase space consists of a vertically bending dipole magnet (bend radius $r=300 \mathrm{~mm}$ ) which bends and disperses the beam onto an aerogel screen from which then promptly emits small angular-spread Cerenkov light that is transported to a Hamamatsu C5680 streak camera having 2 ps resolution. The beam's momentum distribution $\delta p / p$ was resolved using the $R_{36}$ (vertical dispersion) of the dipole and the shearing of the phase space due to the contribution of the transport element $R_{56}$ from the dipole was removed to determine the beam's temporal distribution [14].

The transverse projected emittance is measured using a slit-scan technique where a screen is employed at some point $z_{0}$ with a slit placed upstream of $z_{0}$ and scanned across the beam, to yield a measurement of the divergence distribution associated with each spatial (in $x$ or $y$ ) slice in the beam. The divergence distributions are then combined to calculate the emittance [12].

As mentioned above, PARMELA simulations were performed using the design field profiles for gun and nine-cell L-band normal conducting booster linac as modeled in SUPERFISH, as well the solenoid, per its POISSON model. The beam energy after the gun exit was matched in simulation by measurement of the field strength in the gun and spectrometer measurements. The measured postbooster linac energy was then used to infer simulated booster field strength. In both simulation and measurement all rf structures were run near crest to yield optimal acceleration.

The results for the above-described experiment were compared with PARMELA simulation [17] and good agreement between simulation and measurement is shown, as 


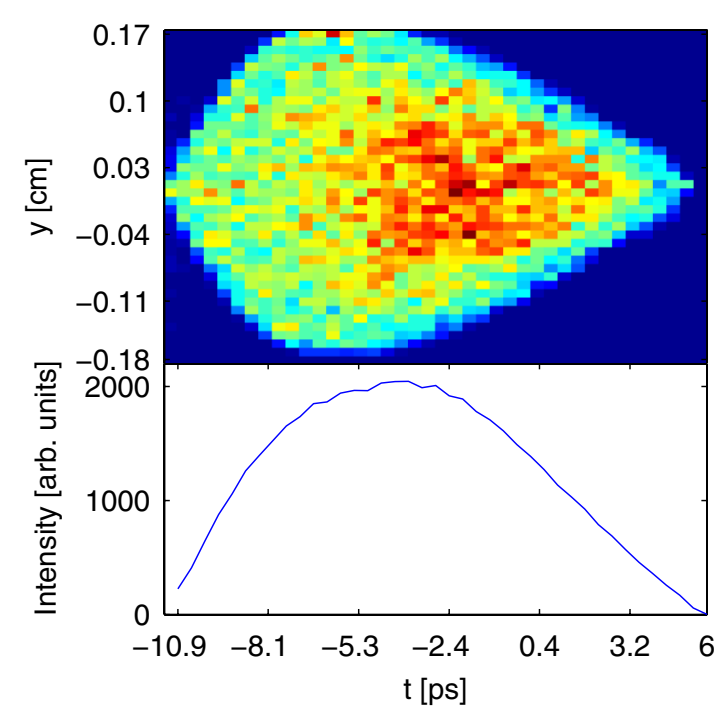

FIG. 1. PARMELA simulation of the beam as seen at the linac entrance. As can be seen the beam assumes an approximately ellipsoidal shape. The antisymmetry is due to image charge effects at the cathode. Below the configuration space plot is the temporal projection of the PARMELA-generated distribution at the linac entrance.

seen below. This comparison is discussed further in the results section.

As noted, before undertaking the experiments PARMELA was used to determine whether or not the PITZ laser and rf gun in combination were capable of producing injected pulses which meet the necessary requirements for ensuring that the self-shaping effect was observable. Working with measured charge production for specific beam shaping apertures, simulations were run to determine if blowout occurs and to what extent. Previous experiments had shown beam size increases from 210 fs full width to 2.5 ps full width, i.e., a total longitudinal growth of around 12 times [9]. Through preliminary simulation it was determined in the PITZ experimental environment that the $750 \mathrm{pC}$ beam of 2.7 ps FWHM Gaussian cut transversely using a $2 \mathrm{~mm}$ beam shaping aperture produces a resulting beam length of 9.9 ps FWHM ellipsoidal distribution, or an increase of around 3.7 times the initial length. As apparent in Fig. 1 the effect of image charge on the electron distribution as generated at the cathode is quite large and in fact, given the $750 \mathrm{pC}$ charge and transverse dimensions, is approximately equal to the accelerating gradient at $50 \mathrm{MV} / \mathrm{m}$, 10 times higher than the image charge field generated in previous blowout experiments [9].

As is shown in Fig. 1 the beam is expected to transform from a Gaussian beam in the longitudinal direction into an approximately ellipsoidal shape, in this case not quite uniformly filled due to both the remanent evidence of the initial Gaussian temporal spread and image charge effects, as were also noted in the proof-of-principle experiments described in Ref. [9]. Furthermore, as shown in Fig. 2, the

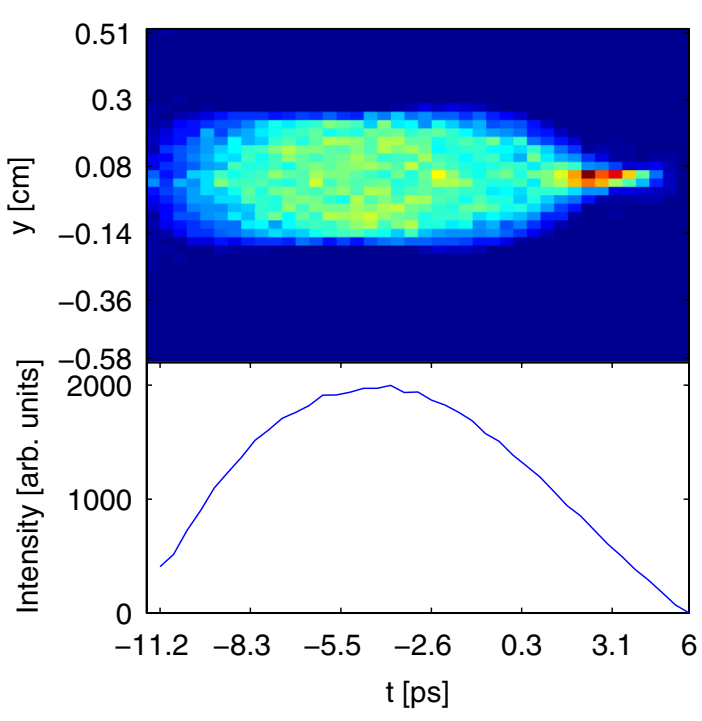

FIG. 2. PARMELA simulation of the beam as seen at the dipole entrance, after secondary acceleration in a booster cavity. As can be seen the beam assumes an ellipsoidal shape, with some distortion at beam rear due to image charge. Below the configuration space plot is the projected temporal distribution from PARMELA at the dipole entrance, showing the approximately half-circle beam profile.

elongated tail is transformed into a hot spot after ponderomotive focusing in the booster linac. The machine was optimized to produce the lowest emittance (consistent with simulation of compensation dynamics) and was accelerated to $12 \mathrm{MeV}$. At this higher energy, the approximately ellipsoidal shape is preserved, providing for optimal emittance compensation.

\section{RESULTS}

The streak camera image from which the longitudinal phase space (LPS) measurement is extracted is shown in Fig. 3. In order to first compare it to the PARMELA model, we ran the simulation up to the dipole entrance, but as the PARMELA model for collective effects is not adequate to describe space-charge effects in a tight radius-of-curvature

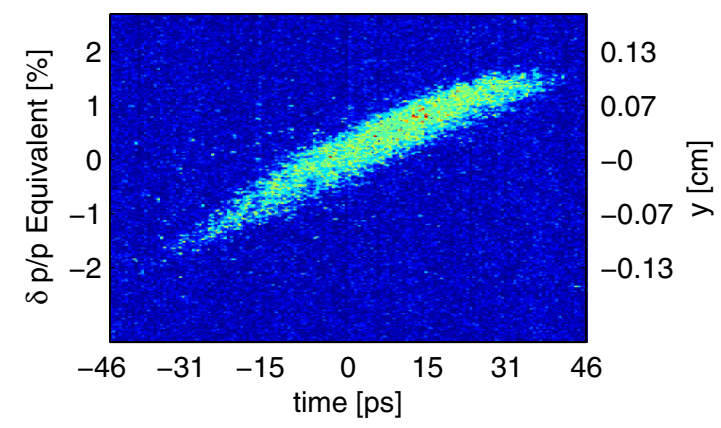

FIG. 3. The raw streak camera data after the dipole as measured using an aerogel screen and a Hamamatsu C5680 streak camera. 


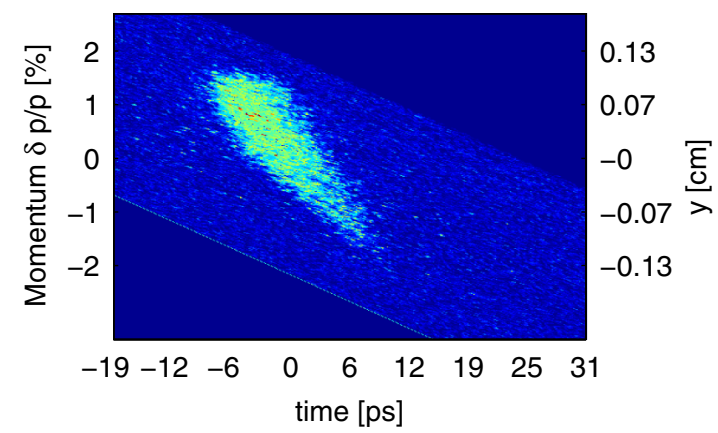

FIG. 4. Streak camera data with the effect of the $R_{56}$ shear removed to provide the longitudinal phase space at the dipole entrance.

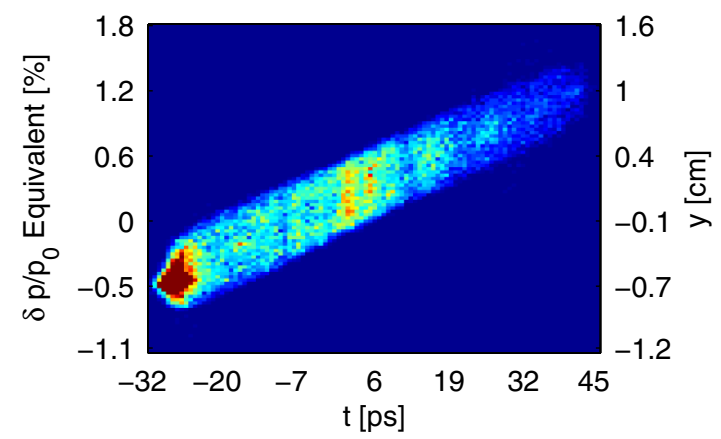

FIG. 5. The simulated PARMELA beam after passage through a dipole, using simple matrix transport methods. While the overall shape is similar to the beam as measured at PITZ, features such as a denser beam head are not reproduced, likely due to lack of inclusion of collective charge effects.

bend, we did not rely on PARMELA for this simulation, but simply used matrix transport to obtain a prediction for the observed beam image in the streak camera. The matrix approach is employed also because of its utility in analyzing the longitudinal phase space measurements. We also note that, despite an effort at focusing the beam optimally, the resolution of the momentum distribution is coarse in both the data and simulation, due to the presence of nonnegligible betatron width in the vertical ( $y$, dispersive) direction.

To establish the existence of an approximately selfshaped ellipsoidal distribution, the streak camera data was analyzed by removal of the $R_{56}$ shear and use of the $R_{36}$ of the dipole to resolve the longitudinal phase space. PARMELA gives a beam length of approximately $14 \mathrm{ps}$ full length and the measurement of the same quantity produced 15.6 ps (see Figs. 4 and 5). Beyond this important quantitative measure, important features in the measured LPS worth noting are the beam displays the expected highly correlated beam chirp due to its self-shaping expansion (well beyond that which may be attributed to possible effects due to rf phasing of the linac) and the asymmetry between the shapes of front and the back of the beam,

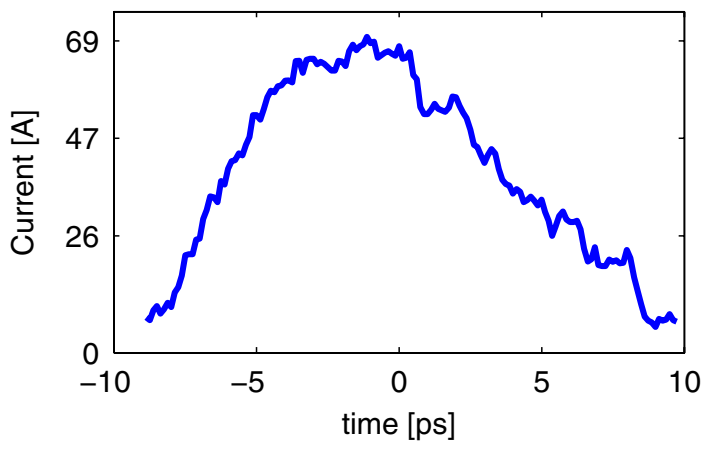

FIG. 6. The longitudinal beam density profile as measured at PITZ. The beam's front (earlier time) in this profile displays the half-circle profile characteristic of self-shaped beams, with distortions due to image charge effects noted at the rear of the beam. This profile is generated after the $R_{56}$ shear is removed.

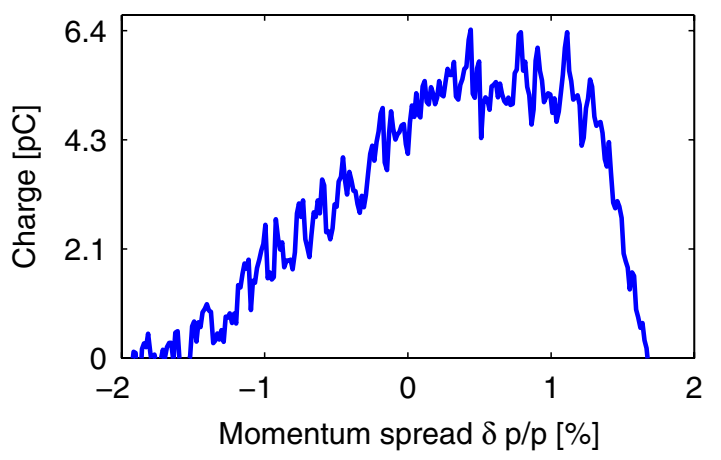

FIG. 7. The momentum distribution of the beam as measured at PITZ.

respectively. This second artifact is indicative of the image charge effects at the cathode. Beyond this nonideal aspect in the measurements due to collective effects, previous measurements [10] had employed a slit to eliminate space-charge effects inside the dispersive section itself. This was not done in the measurements at PITZ, and it can be assumed that some additional measured beam length is due in part to further space-charge induced elongation as well as dispersion errors. Furthermore, the simulation did not take into account field enhanced charge production at the cathode, only the magnitude of charge produced.

After the $R_{56}$ shear was removed, the beam was projected onto the time axis for examination of the beam density. As is shown in Fig. 6, the beam approximates the half-circle profile characteristic of the projection of self-shaped ellipsoidal beams. Further, the momentum distribution was projected from the LPS, as shown in Fig. 7. As expected, for a properly blown-out beam the momentum distribution is sharply defined. In both plots the beam asymmetry attributable to image charge effects at the cathode are evident: the half-circle density profile is 


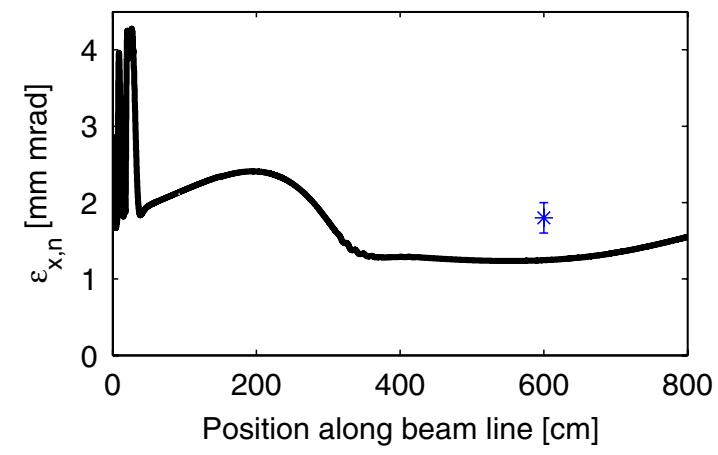

FIG. 8. Simulation of the emittance evolution in PARMELA. The point with error bars indicates the emittance as measured at PITZ.

slightly corrupted, and the momentum distribution generates a low energy tail due to additional deceleration at the back of the beam.

The measured emittance obtained from the slit mask setup was $1.8 \mathrm{mmmrad}$ with the somewhat idealized PARMELA prediction giving $1.4 \mathrm{~mm} \mathrm{mrad}$ at the relevant beam line position (Fig. 8). Nonconformity with simulation results may arise from initial laser nonuniformities, transverse wakefields, and systematic error due to measurement averaging in the presence of the current rf phase fluctuations $[18,19]$. We have already demonstrated good emittance performance, and with the known problems addressed, one may expect to achieve excellent emittance. In this regard we note that, because of the somewhat low energy of the booster in these measurements, the emittance compensation process is not complete, and one can achieve less that $\epsilon_{n}<1.4 \mathrm{~mm} \mathrm{mrad}$ in simulation using further postacceleration. It is interesting to note that, as one might expect, we experimentally obtain higher peak beam current in this alternate beam optimization than in the standard scenario [20], despite employing only $75 \%$ the charge.

\section{CONCLUSIONS}

It has been shown that, even considering some nonideal aspects of the experimental scenario (a smaller ratio of final to initial beam length, and notable image charge effects) self-arrangement of the beam due to space-charge forces can be used to generate well optimized beams. The beam line in use for this experiment was optimized for a different initial beam profile, the standard uniform, longer pulse distribution, but it was still possible to produce a beam displaying the desired self-formed properties. Furthermore, it has been shown that the shape due to space-charge expansion is maintained even through postphotoinjector acceleration. Finally, we note that this is a first test of the blowout regime in the context of lower-field L-band photoinjectors, which will undoubtedly play a major role in future high average power free-electron lasers.
In addition, we find that, given the problems outlined above, reasonable emittance values as well as high current and linearity of the longitudinal phase space makes the blowout regime scenario attractive as a precursor to magnetic compression. This aspect of the blowout regime should be tested, but this is beyond the current capability of the PITZ injector infrastructure. The same LPS advantage can be claimed a priori about compression via velocity bunching. An experiment exploring this possibility could be performed at the SPARC facility in Frascati, which has demonstrated capabilities in blowout regime studies [8] and velocity bunching with emittance compensation [21] in collaboration with the UCLA. It should be noted that in the case of velocity bunching scenario collective effects at low energy can strongly modify the linearity of the LPS distribution.

Further investigation is warranted in the study of cathode image charge effects in simulation including, but not limited to, use of particle-in-cell codes and other beam dynamics codes which have shown promise in more adequately reproducing image charge effects. Future installation of a deflecting cavity in the PITZ beam line will make it possible to directly examine the longitudinal profile of the beam, as well as its LPS, allowing for a more in depth analysis of beam evolution.

[1] B. Carlsten, Nucl. Instrum. Methods Phys. Res. 285, 313 (1989).

[2] L. Serafini and J. B. Rosenzweig, Phys. Rev. E 55, 7565 (1997).

[3] I. Kapchinskii and V. Vladimirskii, in Proceedings of the International Conference on High Energy Accelerators (CERN, Geneva, 1959), p. 274.

[4] S. Chandrasehkar, Ellipsoidal Figures of Equilibrium (Yale University Press, New Haven, CT, 1969), p. 43.

[5] O.D. Kellogg, Foundations of Potential Theory (The Murray Printing Company, Cambridge, MA, 1929), p. 192.

[6] L. Serafini, AIP Conf. Proc. 413, 321 (1997).

[7] O. J. Luiten, S. B. van der Geer, M.J. de Loos, F. B. Kiewiet, and M. J. van der Wiel, Phys. Rev. Lett. 93, 094802 (2004).

[8] J. Rosenzweig, A. Cook, R. England, M. Dunning, S. Anderson, and M. Ferrario, Nucl. Instrum. Methods Phys. Res. 557, 87 (2006).

[9] P. Musumeci, J. T. Moody, R. J. England, J. B. Rosenzweig, and T. Tran, Phys. Rev. Lett. 100, 244801 (2008).

[10] J. T. Moody, P. Musumeci, M.S. Gutierrez, J. B. Rosenzweig, and C.M. Scoby, Phys. Rev. ST Accel. Beams 12, 070704 (2009).

[11] L. Staykov, Ph.D. thesis, University of Hamburg, 2008.

[12] L. Staykov, in Proceedings of the 2007 FEL Conference, Novosibirsk, Russia, 2007), p. 138 [http://accelconf.web .cern.ch/accelconf/f07/INDEX.HTM].

[13] J. Rönsch, Ph.D. thesis, University of Hamburg, 2009. 
[14] J. Rönsch, in Proceedings of DIPAC09, Villigen, Switzerland, 2009), p. 360 [http://dipac09.web.psi.ch/ Proceedings/index.htm].

[15] M. Hänel, Ph.D. thesis, University of Hamburg, 2010.

[16] M. Hänel, in Proceedings of the 2007 FEL Conference, Novosibirsk, Russia, 2007 (Ref. [12]), p. 346.

[17] http://laacg1.lanl.gov/laacg/services/serv_codes.phtml\# parmela.

[18] F. Stephan, in Proceedings of the Physics and Applications of High Brightness Electron Beams, Maui, Hawaii,
2009 [http://pbpl.physics.ucla.edu/HBEB/PlenaryTalks/ STEPHAN.pdf].

[19] M. Krasilnikov, in Proceedings of the Physics and Applications of High Brightness Electron Beams (Ref. [18]).

[20] F. Stephan et al., Phys. Rev. ST Accel. Beams 13, 020704 (2010).

[21] L. Serafini and M. Ferrario, AIP Conf. Proc. 581, 87 (2001). 